\title{
Thermally Induced Charge Reversal of Layer-by- Layer Assembled Single-Component Polymer Films
}

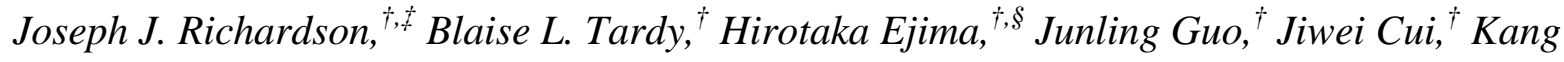

Liang, ${ }^{\dagger, \ddagger}$ Gwan H. Choi, ${ }^{\#}$ Pil J. Yoo, ${ }^{\#}$ Bruno G. De Geest, ${ }^{\perp}$ and Frank Caruso ${ }^{*}, \dagger$

${ }^{\dagger}$ ARC Centre of Excellence in Convergent Bio-Nano Science and Technology, and the Department of Chemical and Biomolecular Engineering, the University of Melbourne, Parkville, Victoria, Australia

${ }^{\#}$ School of Chemical Engineering and SKKU Advanced Institute of Nanotechnology (SAINT),

Sungkyunkwan University, Suwon, 16419, South Korea

${ }^{\perp}$ Department of Pharmaceutics, Ghent University, Ghent, Belgium

KEYWORDS: layer-by-layer assembly, low-fouling, cell adhesion, single-polymer, charge conversion 


\section{ABSTRACT}

Temperature can be harnessed to engineer unique properties for materials useful in various contexts, and has been shown to affect the layer-by-layer (LbL) assembly of polymer thin films and cause physical changes in preassembled polymer thin films. Herein, we demonstrate that exposure to relatively low temperatures $\left(\leq 100^{\circ} \mathrm{C}\right)$ can induce physico-chemical changes in cationic polymer thin films. The surface charge of polymer films containing primary and secondary amines reverses after heating (from positive to negative), and different characterization techniques are used to show that the change in surface charge is related to oxidation of the polymer that specifically occurs in the thin film state. This charge reversal allows for single-polymer LbL assembly to be performed with poly(allylamine) hydrochloride (PAH) through alternating heat/deposition steps. Furthermore, the negative charge induced by heating reduces the fouling and cell-association of PAH-coated planar and particulate substrates, respectively. This study highlights a unique property of thin films which is relevant to LbL assembly and bio-fouling, and is of interest for the future development of thin polymer films for biomedical systems. 


\section{INTRODUCTION}

The layer-by-layer (LbL) assembly of polymers is a useful technique for preparing thin films with various chemical properties and physical characteristics. ${ }^{1,2}$ Each polymer layer is typically on the order of a few nanometers, which allows for thin, highly defined films to be engineered on a variety of substrates. ${ }^{3,4}$ Moreover, the LbL assembled films often retain the functionality of their constituent polymer layers, depending on the materials, technique, and interactions used for assembly. ${ }^{1,5}$ Electrostatic interactions are commonly used to assemble multilayer films, though other mechanisms, including hydrogen bonding, host-guest interactions, and click-chemistry can

also be used. ${ }^{5,6}$ For electrostatic assembly, one polymer is positively charged and the other is negatively charged, therefore making multilayer films from a single charged polymer difficult due to the electrostatic repulsion of like-charged materials. ${ }^{7}$ Single-polymer films are often useful due to the fact that their material properties can be dictated solely by the physico-chemical properties of the individual polymer, such as $\mathrm{pH}$-dependent shrinkage and swelling, without issues such as polymer-polymer reactivity. ${ }^{8}$ Generally, these single-polymer films are prepared by avoiding electrostatic interactions, and can be achieved by layering a hydrogen-bonding sacrificial polymer with the polymer of interest. The polymer of interest can be selectively crosslinked, and then the sacrificial polymer released by a specific trigger, such as a change in pH. ${ }^{8,9}$ A method for making single-polymer multilayer films without sacrificial layers is to use a covalent cross-linker, such as glutaraldehyde, to stabilize layers of polymer containing functional groups, like amines, although in both cases harsh crosslinking chemicals can modify or denature fragile cargo. ${ }^{10-12}$

Herein, we utilize a recently explored property of thin films, where heat can affect the polymer in a thin film differently than the polymer in bulk solution, to facilitate single polymer LbL 
assembly and to engineer specific bio-nano interactions. Previous reports have shown that physical and chemical properties can be different for polymers in a thin film compared with the same polymer in bulk; for example the glass transition temperature of some polymers is significantly lower when assembled into a thin film when compared with the bulk polymer glass transition temperature. ${ }^{13-15}$ A similar phenomenon was recently observed during LbL assembly methods that require heat to remove LbL coated particles from a thermo-responsive immobilizing matrix. ${ }^{7,10,16}$ The act of heating (within or without an immobilizing matrix) caused the cationic polymer poly(allylamine) hydrochloride (PAH) to lose its inherent positive charge and become negative without any polymer desorption. ${ }^{7,10}$ In the current study, heat is similarly used to cause a reversal in the surface charge of cationic thin films composed of primary and secondary amines, and the structural and chemical changes of the polymer and thin film are investigated. Additionally, the reverse in surface charge is utilized to allow the adsorption of additional layers of polymer, whereby repeated heating/deposition steps allow for the LbL assembly of single-component polymer films (Figure 1). This assembly process allows for LbL films, capsules and replica particles to be prepared from only PAH, while the reversal in surface charge also reduces protein adsorption onto planar films and reduces the binding of particles coated in the heat-prepared and treated LbL films. 


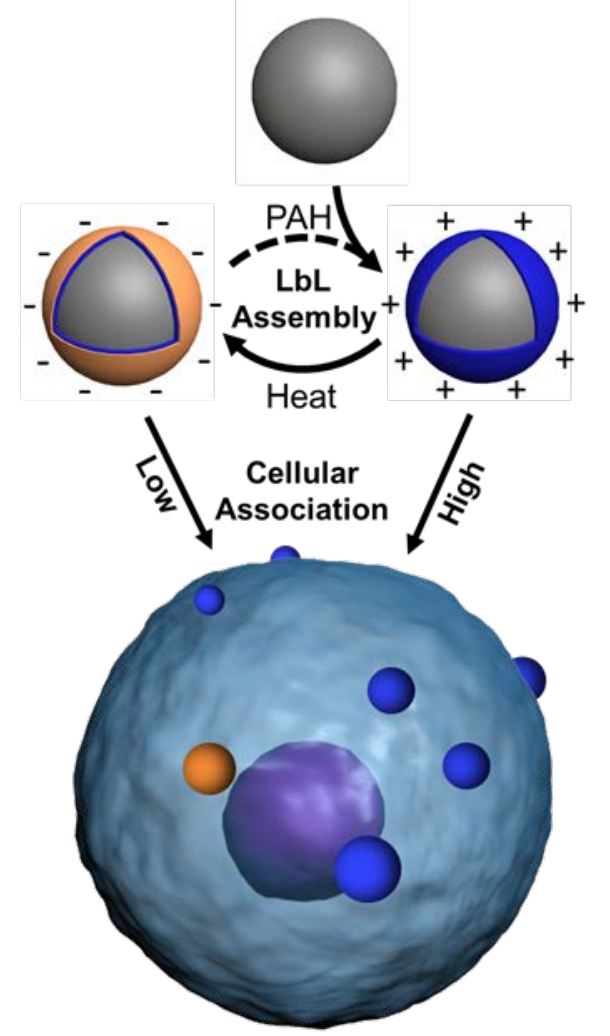

Figure 1. LbL assembly of $\mathrm{PAH}$ on particles. After coating particulate templates with $\mathrm{PAH}$ (blue), heat can be used to reverse the surface charge (orange) to allow for repeated deposition cycles. A final heating process also decreases the fouling and cell-adhesion of core-shell particles.

\section{EXPERIMENTAL SECTION}

2.1 Film Deposition of PAH. PAH (15 kDa or $58 \mathrm{kDa}$ from Sigma) was dissolved at $1 \mathrm{mg} \mathrm{mL}^{-1}$ in $0.5 \mathrm{M} \mathrm{NaCl}$ and the $\mathrm{pH}$ was adjusted to 6.5. For planar substrates, the substrate was first cleaned with Piranha solution (3:7 ratio of $30 \% \mathrm{H}_{2} \mathrm{O}_{2} / 98 \% \mathrm{H}_{2} \mathrm{SO}_{4}$ ) for $1 \mathrm{~min}$, rinsed with ultrapure water, and dried in a stream of nitrogen. [Caution! Piranha solution reacts violently with organic material and should be handled carefully.] The substrate was then immersed in the PAH solution for $15 \mathrm{~min}$, followed by three rinsing steps in ultrapure water. For particulate 
substrates, the particles were dispersed in polymer solution for $15 \mathrm{~min}$, followed by three cycles of centrifugation $(1000 \mathrm{~g})$ and supernatant removal, and redispersion in ultrapure water. The substrates were then immersed (planar) or dispersed (particulate) in ultrapure water and placed in an oven set to $100{ }^{\circ} \mathrm{C}$ for $15 \mathrm{~min}$, followed by immersion or dispersion into ultrapure water at room temperature. LbL assembly was accomplished by then repeating the deposition and heating steps. The calcined calcium carbonate $\left(\mathrm{CaCO}_{3}\right)$ template particles were dissolved using $50 \mathrm{mM}$ sodium acetate ( $\mathrm{pH} 4)$, while the silica $\left(\mathrm{SiO}_{2}\right)$ template particles were dissolved using $5 \mathrm{M} \mathrm{HF}$. [Caution! HF is extremely dangerous and should be handled carefully!]

2.2 Characterization. $\zeta$-potential was measured by a Malvern Zetasizer Nano ZS, using the Malvern software. Samples were dispersed in ultrapure water before measuring. Proton NMR $\left({ }^{1} \mathrm{H}-\mathrm{NMR}\right)$ was carried out using a $400 \mathrm{MHz}$ Varian INOVA system at $25^{\circ} \mathrm{C}$. The spin-lattice relaxation time (T1) was determined via a conventional inversion recovery pulse sequence, $90^{\circ}-\mathrm{t}-180^{\circ}$, under deuterium lock mode. Spectra were referenced to residual proton resonances of the deuterated solvent $\left(\mathrm{D}_{2} 0\right)$. Chemical shifts are reported as parts per million (ppm). Raman spectra were gathered using a Horiba confocal Raman microscope with a 20× objective lens. Fourier transform infrared (FTIR) spectra were recorded using a Varian 7000 FTIR spectrometer with a Specac MKII Golden Gate single-reflectance diamond ATR attachment equipped with KRS-5 optics and a heated top plate maintained at $25{ }^{\circ} \mathrm{C}$. Flow cytometry measurements were performed using a Partec CyFlow Space flow cytometer at an excitation wavelength of 488 and analyzed using FlowJo v8.7. To calculate the adsorbed mass, quartz crystal microbalance (QCM) chips (gold-coated) were analyzed with a home-built QCM. Contact angles were measured using a Model 200 standard goniometer (Ramé-Hart, USA). All contact angles were measured with a $10 \mu \mathrm{L}$ water droplet at $25^{\circ} \mathrm{C}$. The recorded contact angles are averages of three measurements 
made on different areas of the surface. Microscope images were obtained using an inverted Olympus IX71 microscope equipped with a $60 \times$ objective lens. A CCD camera was mounted on the left-hand port of the microscope and fluorescence images were illuminated with an X-Cite module with fluorescein isothiocyanate (FITC) filters. Atomic force microscopy (AFM, Dimension 3100, Veeco, USA) measurements were conducted in tapping mode under ambient conditions. Transmission electron microscopy (TEM) images and energy dispersive X-ray spectroscopy (EDX) profiles were acquired by using a FEI Tecnai TF20 instrument equipped with a SDD detector for TEM. The electron beam was accelerated with an operation voltage of 200 kV. Differential scanning calorimetry (DSC) was measured using a DSC 6220N (SII Nano Technology, Inc.) at a heating rate of $10{ }^{\circ} \mathrm{C} \mathrm{min}^{-1}$ in air or under a flow of nitrogen (50 $\mathrm{mL} \mathrm{min}^{-}$ ${ }^{1}$ ). PAH powders of ca. $5 \mathrm{mg}$ were used for measurements.

2.3 Cellular Association and Protein Fouling. Cellular association studies were performed by plating out triplicates of 50000 JAWS II cells per well in Dulbecco's Modified Eagle’s Medium in 24-well plates. Core-shell particles were then added into the wells with a particle-to-cell ratio of 10:1 or 100:1 and incubated for $2 \mathrm{~h}$. After incubation free particles were washed off using phosphate buffered saline (PBS), and then trypsin was added, the cells were incubated for 10 min and then the free cells were washed into PBS three times by centrifuging at $500 \mathrm{~g}$ for $5 \mathrm{~min}$. The washed cells were then placed into flow cytometry tubes and at least 3000 cells per well were analyzed using flow cytometry. Protein fouling was performed by immersing the PAH-coated QCM chips into $100 \%$ fetal bovine serum and incubating them at $37{ }^{\circ} \mathrm{C}$ for $15 \mathrm{~min}$. The chips were then washed in ultrapure water, dried in a nitrogen stream, and the frequency recorded. 


\section{RESULTS AND DISCUSSION}

3.1. Characterization of Thermally Induced Charge Reversal. Previous reports on heating PAH multilayers prompted us to investigate the effect of heat on cationic polymers. PAH-coated $\mathrm{SiO}_{2}$ template particle suspensions were heated at $100{ }^{\circ} \mathrm{C}$ for $15 \mathrm{~min}$, and similar to what was shown in recent studies, the $\zeta$-potential permanently became negative without any recovery (Figure 2A). ${ }^{7,10}$ Temperatures lower than $100{ }^{\circ} \mathrm{C}$ also resulted in significant changes in the $\zeta$ potential, leading to negatively charged $\mathrm{PAH}$ films even at temperatures as low as $50{ }^{\circ} \mathrm{C}$ (Figure 2B). Charge changes at these lower temperatures may have implications for fields where the temperature is raised above room temperature, such as catalysis and drug delivery. Pre-heated bulk PAH had a similar $\zeta$-potential $(+35 \mathrm{mV})$ in comparison to non-heated PAH (+45 mV) after layering, suggesting that the polymer in bulk does not respond the same way to heat compared to when it is in a thin film, as has been demonstrated previously. ${ }^{13}$ Additionally, it has been shown that even when PAH is in a film, the thickness of the film plays a significant role in determining the effects of heat, with the substrate contributing as a catalyst for thinner films. ${ }^{15}$ The molecular weight of PAH (15 kDa and $58 \mathrm{kDa}$ ) did not make a significant difference to the charge reversal, and also the presence of salt did not make a difference, as desalted poly(allyl amine) ${ }^{17}$ showed a similar charge reversal, both of which further suggested that the charge reversal was primarily dependent on the polymer being assembled into a thin film. The $\mathrm{pH}$ of the solution, however, did play an important role, with protonated $\mathrm{PAH}(\mathrm{pH}$ 4) yielding the smallest change in $\zeta$-potential ( $\sim 30 \mathrm{mV})$, and deprotonated PAH ( $\mathrm{pH} 10)$ yielding the largest change $(\sim 75 \mathrm{mV})$, while an intermediary change of $\sim 60 \mathrm{mV}$ was found after heating at neutral $\mathrm{pH}$ once the samples were returned to neutral $\mathrm{pH}$ through repeated wash steps. This suggested that a basic, deprotonated form was more favorable for the $\zeta$-potential change. To confirm this hypothesis, other polymers 
containing primary (poly-L-Lysine, PLL), secondary and tertiary (poly-L-Histidine, PHIS), and quaternary (polydiallyldimethylammonium chloride, PDADMAC) amines were deposited on $\mathrm{SiO}_{2}$ template particles and heated at $100{ }^{\circ} \mathrm{C}$ for $15 \mathrm{~min}$. The $\zeta$-potentials for the polymer thin films composed of primary and secondary amines became negative after heating, while the films composed of polymers containing quaternary amines remained positively charged, supporting the hypothesis that deprotonated amines with free electrons were required for the charge-reversal (Figure 2C). For all of the polymers containing primary and secondary amines, a second layer of polymer could be deposited after the heat treatment, causing a second charge reversal (from negative to positive) (Figure 2C). 

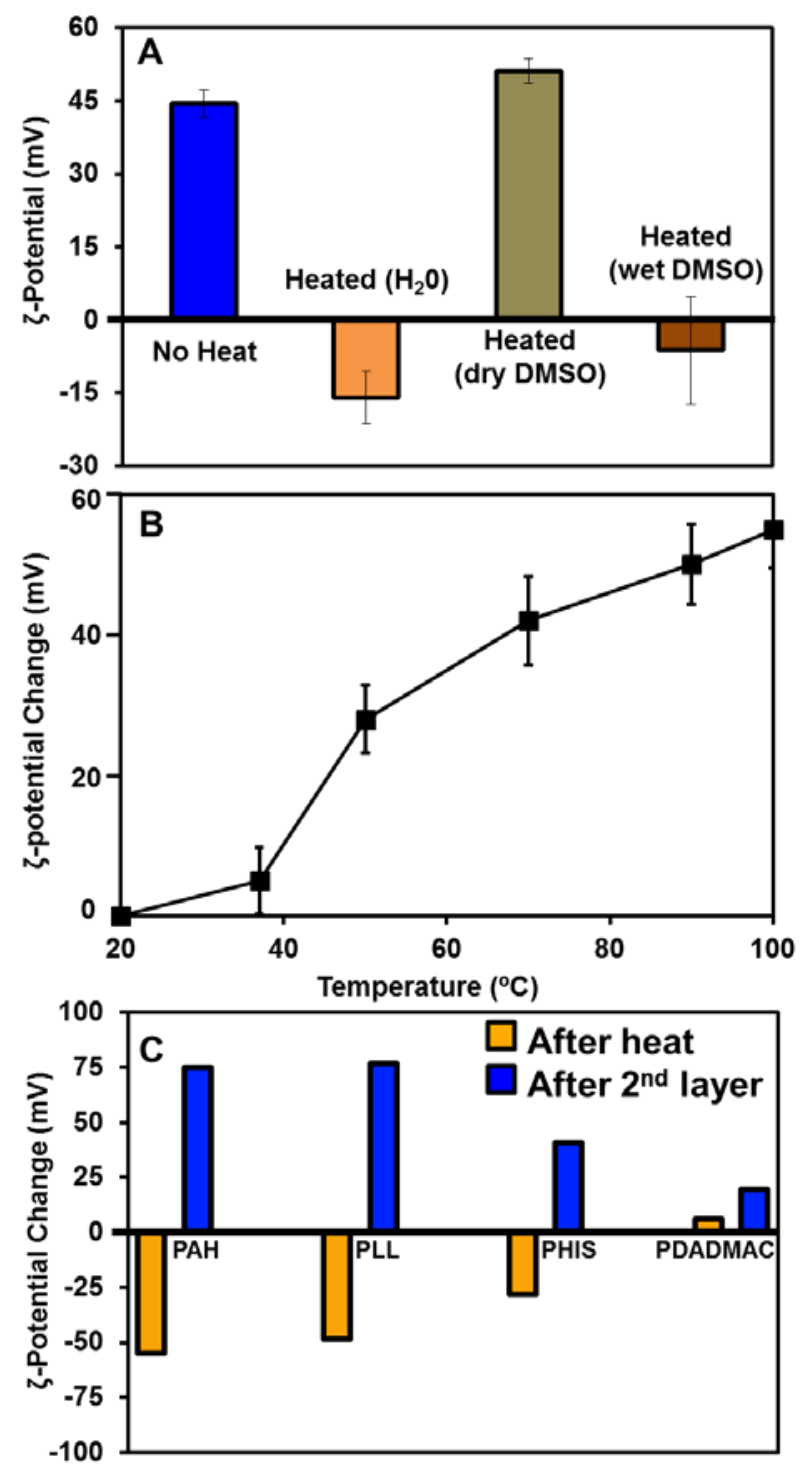

Figure 2. Effect of heating on different polymer films. (A) $\zeta$-potential of PAH thin films deposited on $\mathrm{SiO}_{2}$ measured in different solvents. (B) Change in $\zeta$-potential resulting from 15 min incubation at different temperatures for PAH thin films. (C) Change in $\zeta$-potential after heating at $100{ }^{\circ} \mathrm{C}$ for 15 min for PAH, PLL, PHIS and PDADMAC thin films after heating (orange) and after the deposition of a second polymer layer (blue).

The charge reversal of the polymer suggested that the polymer was being oxidized, and it was confirmed that an abundance of oxygen, $\mathrm{O}_{2}$, was necessary to induce the change to a negative $\zeta$ - 
potential. PAH-coated $\mathrm{SiO}_{2}$ particles did not change $\zeta$-potential after being heated in dry dimethyl sulfoxide (DMSO) (Figure 2); however, doping the DMSO with $\mathrm{H}_{2} \mathrm{O}\left(9: 1, \mathrm{v} / \mathrm{v}\right.$ ) or $\mathrm{O}_{2}$ (by bubbling oxygen through the DMSO for $10 \mathrm{~s}$ ) before heating gave final $\zeta$-potentials of $-7 \mathrm{mV}$ and $-1 \mathrm{mV}$, respectively. Differential scanning calorimetry (DSC) confirmed that the presence of oxygen was critical in the process, as it took higher temperatures to change the heat flow in $\mathrm{N}_{2}$ when compared to air (Figure S1). Energy dispersive X-ray spectroscopy (EDX) was used to determine the nitrogen and carbon contents of the films before and after heating, and it was found that the nitrogen to carbon atomic ratio was roughly 1:3 before heating and 1:4 after heating (Figure S2). This ratio change was identical to the values observed in previous work on the oxidation of PAH films. ${ }^{18}$ In combination with previously reported experiments, ${ }^{13,15}$ these data suggest that the charge reversal is due to oxidation, and moreover that the thin film state is crucial for this process as it is surface-confined.

Previous studies have shown that the brief heat treatment of LbL capsules can result in capsule shrinking or swelling, primarily from solvent exclusion resulting in either densification or electrostatic repulsion, depending on the film constituents. ${ }^{19-23}$ However, the chemical changes of the polymers themselves had not been investigated. Colloidal mesoporous $\mathrm{SiO}_{2}(110 \mathrm{~nm})^{24}$ was used to adsorb enough PAH to be on the lower limit of resolution for proton nuclear magnetic resonance $\left({ }^{1} \mathrm{H}-\mathrm{NMR}\right)$, allowing NMR to be performed on the suspension, as has similarly been performed in previous reports. ${ }^{25,26}$ An NMR spectrum nearly identical to the literature was seen for the non-heated PAH, with the peak at $3.5 \mathrm{ppm}$ corresponding to the protons from the carbon directly adjacent to the amine, while the peaks from 0.8 to $1.6 \mathrm{ppm}$ correspond to the carbon backbone. ${ }^{27}$ The chemical modification of the polymer from the thermal treatment was apparent from the decrease in the intensity of the peak at $1.6 \mathrm{ppm}$ and the appearance of a new peak at 
$2.25 \mathrm{ppm}$, with the downfield shift potentially due to the presence of oxygen in the polymer (Figure 3A). Nevertheless, the most striking observation is that, similar to the EDX experiments, the ratio of carbon to nitrogen largely increased. Bulk PAH heated in solution was similar to the unheated PAH thin film, further confirming the necessity of having the polymer in the thin film state (Figure S3). Raman spectroscopy and Fourier transform infrared spectroscopy (FTIR) further suggested the conversion of the primary amines of PAH into other functional groups and potential cross-linkers through thermal oxidation (Figures 3B and S1). A previous study on the chemical oxidation of PAH proposed possible chemical structures induced by oxidation (reproduced in Figure S4) and due to the negative charge of the heated PAH it is likely that carbon based acids were being formed. ${ }^{18}$ Similar to the NMR study, Raman spectroscopy required the use of a porous particulate substrate to adsorb sufficient PAH to be at the lower detectable limit. In this instance, poly(styrene sulfonate) (PSS)-stabilized calcium carbonate $\left(\mathrm{CaCO}_{3}\right)$ particles were used, as they can adsorb higher quantities of polymer than $\mathrm{SiO}_{2}{ }^{28}$ The peak at $1450 \mathrm{~cm}^{-1}$ corresponds to the carbon backbones of both polymers. ${ }^{29}$ The peaks at 1130 $\mathrm{cm}^{-1}$ correspond to the sulfonate group, and the two small peaks around $1000 \mathrm{~cm}^{-1}$ and the broad peak at $1600 \mathrm{~cm}^{-1}$ correspond to the aromatic portion of PSS, and the double bonds, respectively. ${ }^{30}$ The peaks at $800 \mathrm{~cm}^{-1}$ (N-H rock), $1040 \mathrm{~cm}^{-1}$, and $1330 \mathrm{~cm}^{-1}$ (C-N stretch) correspond to the presence of the primary amine of PAH. ${ }^{31}$ Besides the peak intensity decrease of the primary amine at $1040 \mathrm{~cm}^{-1}$ and minor peak shifts after heating, new peaks appeared at $1080 \mathrm{~cm}^{-1}$ and $1190 \mathrm{~cm}^{-1}$, which is a region common for N-O bonds such as nitro and amide compounds, and also for esters and ethers. For the FTIR spectrum of the unheated PAH film, the broad peak around $3500 \mathrm{~cm}^{-1}$ corresponds to the primary amine N-H stretching, the peaks around $2850 \mathrm{~cm}^{-1}$ and $2950 \mathrm{~cm}^{-1}$ correspond to the alkyl C-H stretch, the bend at $1620 \mathrm{~cm}^{-1}$ to $\mathrm{N}-\mathrm{H}$, the 
peak at $1550 \mathrm{~cm}^{-1}$ from in-plane $\mathrm{N}-\mathrm{H}$ bending, and the peak at $1300 \mathrm{~cm}^{-1}$ to C-N stretching. ${ }^{32-34}$ The broad peak around $1450 \mathrm{~cm}^{-1}$ is due to the carbon backbone of PAH. After heating, a decrease (though not disappearance) in the amine peaks from $1300-1650 \mathrm{~cm}^{-1}$ can be seen, along with the appearance of new peaks in the $1800-2800 \mathrm{~cm}^{-1}$ region. These data correlated well with the Raman spectrum potentially showing the appearance of $\mathrm{N}-\mathrm{O}$ containing functional groups and other oxygen containing groups that are typically found in this region (Figure 3C). Absorbance peaks from the backbone $\left(2850 \mathrm{~cm}^{-1}\right.$ and $2950 \mathrm{~cm}^{-1}$, corresponding to the alkyl C-H stretch and $1450 \mathrm{~cm}^{-1}$, corresponding to the carbon backbone) appear to be largely unaffected in both spectra, reinforcing the hypothesis that the process may principally involve oxidation of the amine groups, similar to what was concluded in a previous report on the oxidation of PAH. ${ }^{18}$ The NMR, Raman, and FTIR results confirmed that chemical changes took place when the thin films of PAH were heated. 

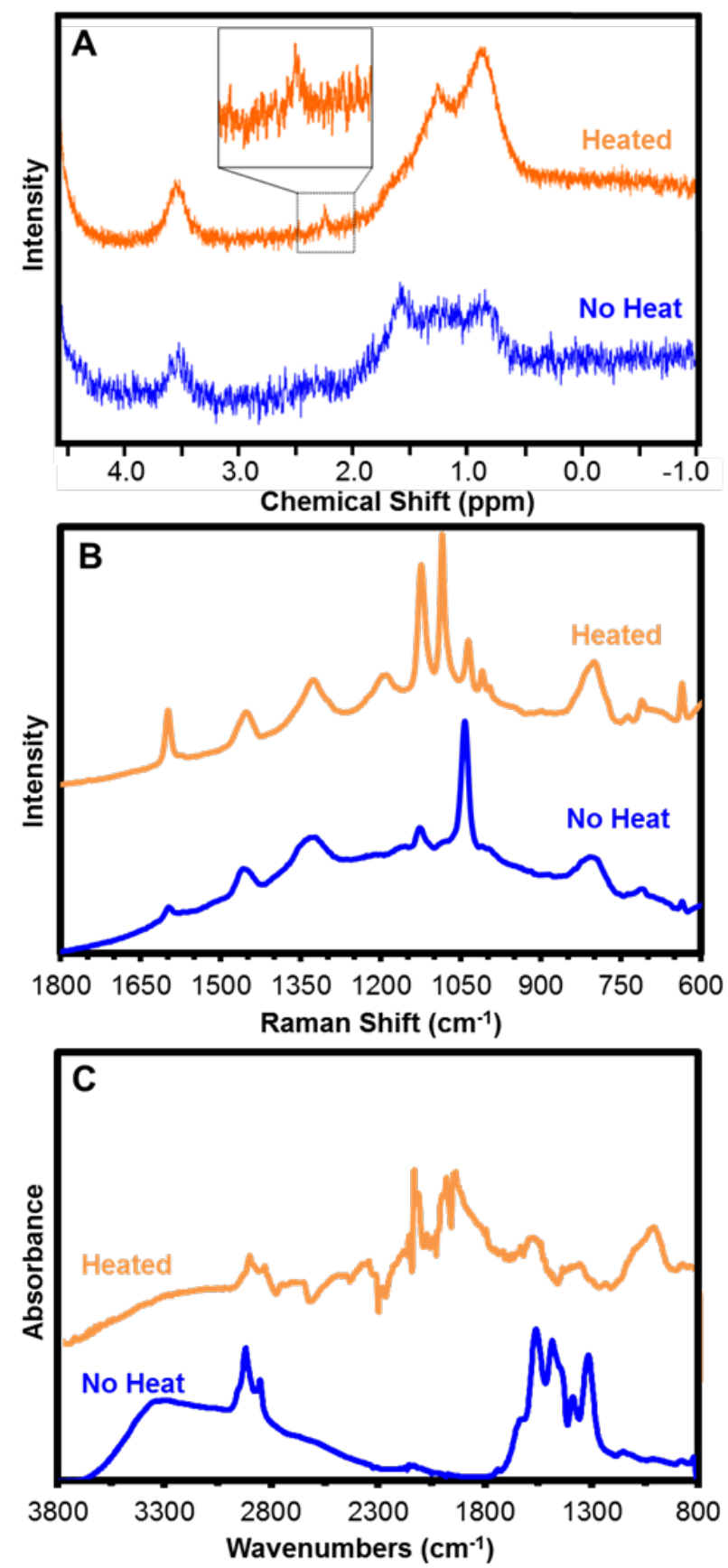

Figure 3. Physico-chemical changes to PAH thin films before (blue) and after (orange) thermal treatment at $100{ }^{\circ} \mathrm{C}$. PAH films measured with: (A) ${ }^{1} \mathrm{H}-\mathrm{NMR}$; (B) Raman spectroscopy; (C) FTIR spectroscopy. 
3.2. Layer-by-Layer Assembly of Single-Component Polymer Films. The evidence that the chemical structure of the polymer changed at elevated temperatures had various implications. The thermally induced charge reversal made it possible to assemble subsequent layers of PAH both onto particulate and planar substrates (Figure 4A, B and Figure S1). Self-assembly steps with intermittent heating steps allowed for linear film growth on both particulate templates and planar substrates, as measured by the fluorescence increase resulting from PAH modified with fluorescein isothiocyanate (PAH-FITC) and quartz crystal microbalance (QCM), respectively. During layer assembly and heating, the contact angle of water on the film changed from $\sim 65^{\circ}$ to $\sim 55^{\circ}$ after the first heat step (Figure S5). These contact angles are consistent with the standard charge reversal of electrostatically assembled LbL films although increased polymer packing with each layer may also partially contribute to this change. ${ }^{35}$ Atomic force microscopy (AFM) measurements showed that the root-mean squared (rms) roughness of a heated $(0.22 \mathrm{~nm})$ and non-heated (0.24 nm) PAH film were similar (Figure S6), which confirmed that the change in surface charge was due to chemical changes rather than polymer spreading or clumping. QCM demonstrated that no polymer was lost during the heating steps (Figure S7), making it likely that the amine groups already interacting with the substrate were not oxidized due to the combined reasons that protonated amines are less likely to oxidize and because of the electrostatic stabilization of being coupled with a negatively charged surface. AFM on scratched planar PAH films yielded a layer thickness of $\sim 0.7 \mathrm{~nm}$ for the first three layers (Figure S8), which is in agreement with PAH layer thickness reported in the literature. ${ }^{1}$ After depositing eight layers of PAH-FITC on $\mathrm{SiO}_{2}$ particles, capsules were formed by removing the template particle core with HF (Figure 4C, D). In contrast, no capsules were formed when the thermally induced charge reversal was avoided after each deposition step. Because of the gradual change in surface charge, 
aggregation became a factor during the heating steps, with sonication aiding in redispersion (Figure S9). The capsules appeared much denser than traditional LbL assembled capsules, such as PAH/PSS capsules, under optical microscopy ${ }^{21}$; however, direct measurement of the shell thickness by AFM was not possible, as the capsules did not collapse. This rigidity can arise from a combination of thermal shrinking and densification, which happen, not during the initial heating process, but during the subsequent layering and heating processes, as shown in previous studies. ${ }^{21}$ Similarly, replica particles could be prepared by adding PAH-FITC to calcined $\mathrm{CaCO}_{3}$ particles via LbL assembly with intermittent heating steps, followed by template removal with sodium acetate (50 mM, pH4) (Figure 4E, F). It was even found that one deposition/heat cycle was enough to assemble PAH replica particles from calcined $\mathrm{CaCO}_{3}$ particles (Figure S10). PAH (and PAH-FITC) heated in the bulk solution were not capable of forming capsules or particles in any of the scenarios mentioned above, which is in line with previous studies that have found significant differences in thin film and bulk properties of polymers. ${ }^{13-15}$ 

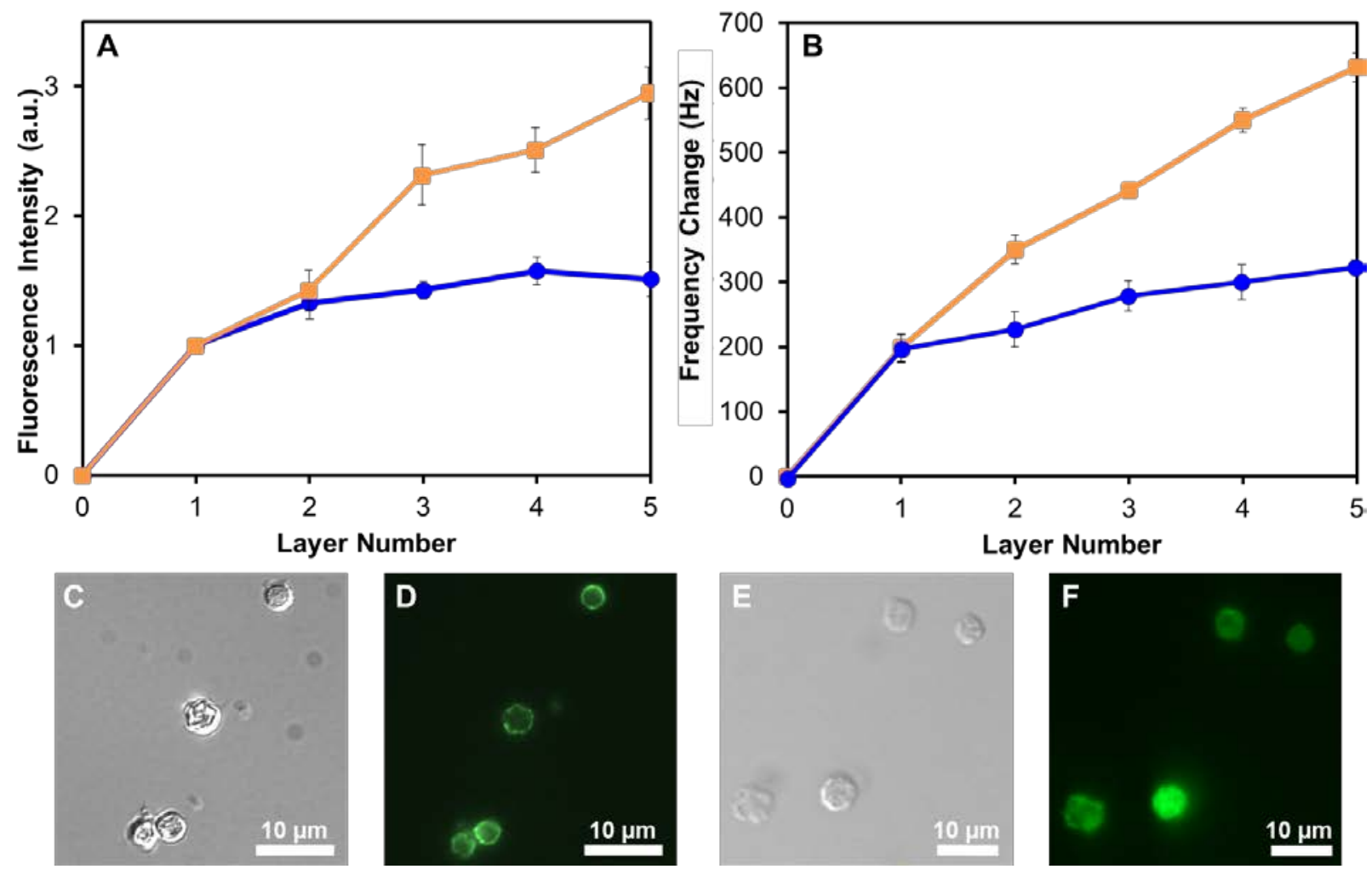

Figure 4. LbL assembly of single-component PAH films. (A) fluorescence increase during the assembly of five PAH-FITC layers on $\mathrm{SiO}_{2}$ particles, as measured by flow cytometry (blue circles - non-heated, orange squares - heated). (B) frequency change during the assembly of five PAH layers on gold substrates, as measured by QCM (blue circles - non-heated, orange squares - heated). (C) bright field image of eight layer PAH capsules prepared from $\mathrm{SiO}_{2}$ particles (3.6 $\mu \mathrm{m}$ in diameter), and (D) corresponding fluorescence image. (E) bright field image of eight-layer PAH-FITC replica particles prepared from calcined $\mathrm{CaCO}_{3}$ particles (5 $\mu \mathrm{m}$ in diameter), and (F) corresponding fluorescence image.

\subsection{Cellular Binding and Protein Fouling of Thermally Charge-Reversed Films. The}

chemical changes exhibited in the thin film state were not just useful for promoting singlecomponent LbL assembly, but could also be harnessed for specific applications. A related phenomenon to the self-assembly of like-charged polymers on a substrate is the spontaneous 
adsorption of biological materials, such as proteins or cells, to polymer films. Because the cell membrane and many proteins are negatively charged, negatively charged materials generally show decreased protein adsorption, or "fouling", and immune responses in comparison to positively charged materials. ${ }^{36-39}$ Hydrated and neutral polymers are even lower-fouling than negatively charged polymers and have been shown to prevent protein adsorption and subsequent cell uptake in vitro and in vivo. ${ }^{24,40,41}$ Due to the relatively high-fouling nature of $\mathrm{PAH}^{42}$ and the fact that modified PAH is FDA approved as sevelamer (in a drug Renagel ${ }^{\circledR}$ ), we investigated whether heating changed the fouling properties and cellular binding of PAH thin films and coreshell particles, respectively. QCM results showed that the heated, planar PAH LbL films had a much lower serum-protein fouling than non-heated PAH films (Figure 5A). Similarly, $1 \mu \mathrm{m}$ particles coated with eight layers of PAH had far lower cell-association with mouse dendritic cells, JAWS II, after heating, when compared to non-heated films (Figures 5B and S11). For the planar studies, there was a five-fold reduction in fouling for heated films compared to non-heated films. For the particle studies, the decrease in cell-association was slightly less, at roughly a three-fold decrease after heating for cell to particle ratios of both 100:1 (Figure 5B) and 10:1 (Figure S11). The overall decrease in the fouling and cell-association of the heated PAH LbL films highlights an alternative approach for engineering materials to minimize biological responses. When combined with the temperature sweep results in Figure 1, it also suggests that some primary amines will undergo weak oxidation in vivo. In fact, after $1 \mathrm{~h}$ at $37{ }^{\circ} \mathrm{C}$ the $\zeta$ potential dropped by $\sim 35 \mathrm{mV}$, suggesting that the room temperature characterization of materials destined for in vivo applications might not accurately reveal the actual physico-chemical properties of the materials in vivo. 

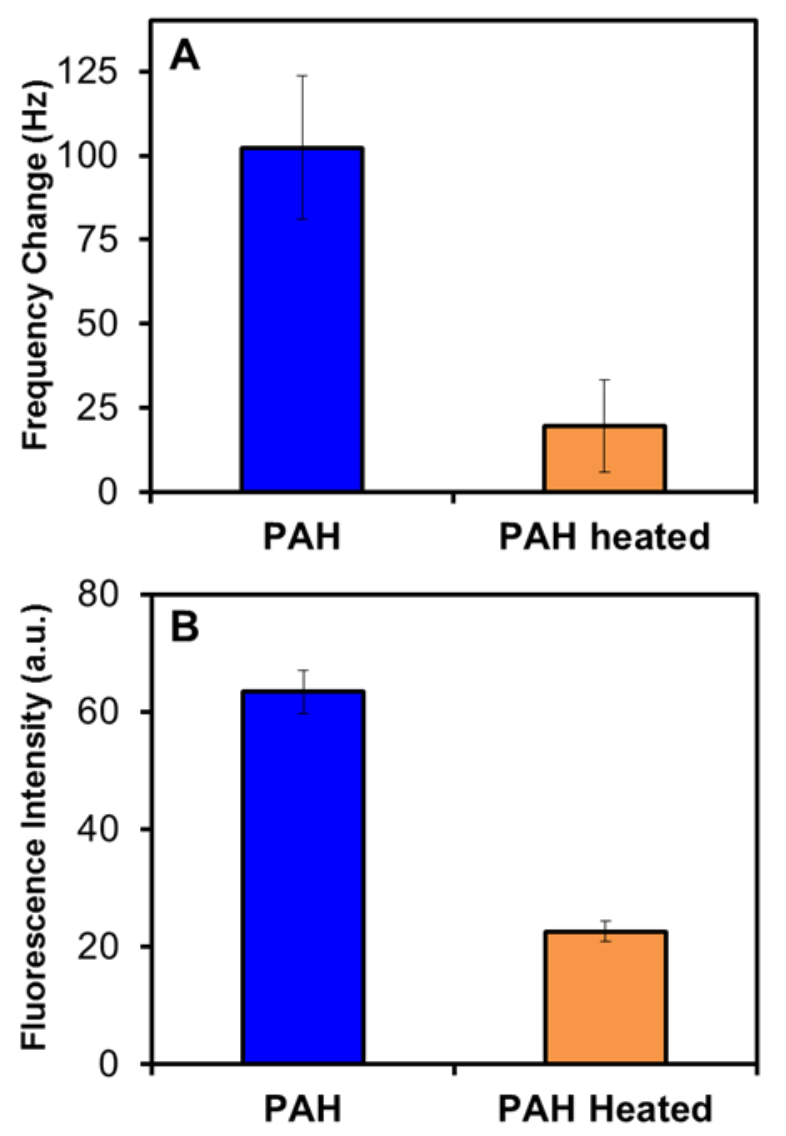

Figure 5. Biological interactions of PAH LbL films and particles. (A) Frequency change after exposure to serum for 15 min, as measured by QCM. (B) Average fluorescence intensity for JAWS II cells, as measured by flow cytometry after incubation with eight layer PAH core-shell particles $(1 \mu \mathrm{m}$ in diameter) for $2 \mathrm{~h}$ at a 100:1 particle-to-cell ratio. The significance level between the two data points in $\mathrm{A}$ was $\mathrm{p}<0.1$, and in $\mathrm{B} \mathrm{p}<0.01$.

\section{CONCLUSION}

We demonstrated the physical and chemical changes arising from heating polymer thin films based on PAH, and how these changes can be harnessed for specific applications. It was shown that heat caused chemical changes in the primary amines of PAH thin films, and that this change was dependent on the solution properties. NMR, FTIR, and Raman spectroscopy demonstrated 
the molecular changes of $\mathrm{PAH}$, while microelectrophoresis showed the charge reversal of the heated PAH. This charge reversal was then used for single-polymer LbL assembly for the formation of capsules and replica particles. Finally, the fouling properties and cell-association behavior of the films and particles were investigated, and it was shown that heating reduces the protein fouling and cell association of PAH thin films. It was shown that similar changes can occur at physiological temperatures over a prolonged period of time, suggesting that the material properties ( $\zeta$-potential, degradation, fouling, etc.) of drug delivery systems should be investigated after prolonged exposure to physiological temperature, rather than at room temperature. Furthermore, short periods of heat could be a way to engineer reduced-fouling properties into amine-containing drug-delivery systems.

\section{ASSOCIATED CONTENT}

Supporting Information. Materials characterization including DSC, EDX, NMR, contact angle, AFM, microscope images, and cell uptake are in the Supporting Information. This material is available free of charge via the Internet at http://pubs.acs.org.

\section{AUTHOR INFORMATION}

\section{Corresponding Author}

*E-mail: fcaruso@unimelb.edu.au

\section{Present Addresses}

${ }^{\ddagger}$ J. J. Richardson, K. Liang, CSIRO Manufacturing, Private Bag 10, Clayton South, Victoria 3169, Australia 
${ }^{\S}$ H. Ejima, Institute of Industrial Science, The University of Tokyo, Tokyo, Japan

\section{Author Contributions}

The manuscript was written through contributions of all authors. All authors have given approval to the final version of the manuscript.

\section{ACKNOWLEDGMENT}

This research was conducted and funded by the Australian Research Council (ARC) Centre of Excellence in Convergent Bio-Nano Science and Technology (project number CE140100036). This work was also supported by the ARC Council under the Australian Laureate Fellowship (F.C., FL120100030) and Discovery Project (F.C., DP130101846) schemes. This work was performed in part at the Materials Characterisation and Fabrication Platform (MCFP) at the University of Melbourne and the Victorian Node of the Australian National Fabrication Facility (ANFF).

\section{REFERENCES}

1. Richardson, J. J.; Björnmalm, M.; Caruso, F. Technology-Driven Layer-by-Layer Assembly of Nanofilms. Science 2015, 348, aaa2491.

2. Decher, G. Fuzzy Nanoassemblies: Toward Layered Polymeric Multicomposites. Science 1997, 277, 1232-1237.

3. $\quad$ Ariga, K.; Hill, J. P.; Ji, Q. Layer-by-Layer Assembly as a Versatile Bottom-Up Nanofabrication Technique for Exploratory Research and Realistic Application. Phys. Chem. Chem. Phys. 2007, 9, 2319-2340.

4. $\quad$ Decher, G.; Hong, J. D.; Schmitt, J. Buildup of Ultrathin Multilayer Films by a SelfAssembly Process: III. Consecutively Alternating Adsorption of Anionic and Cationic Polyelectrolytes on Charged Surfaces. Thin Solid Films 1992, 210/211, 831-835.

5. $\quad$ Ariga, K.; Yamauchi, Y.; Rydzek, G.; Ji, Q.; Yonamine, Y.; Wu, K. C.-W.; Hill, J. P. Layer-by-Layer Nanoarchitectonics: Invention, Innovation, and Evolution. Chem. Lett. 2014, 43, 36-68. 
6. Zhang, X.; Chen, H.; Zhang, H. Layer-by-Layer Assembly: From Conventional to Unconventional Methods. Chem. Commun. 2007, 26, 1395-1405.

7. $\quad$ Richardson, J. J.; Liang, K.; Kempe, K.; Ejima, H.; Cui, J.; Caruso, F. Immersive Polymer Assembly on Immobilized Particles for Automated Capsule Preparation. Adv. Mater. 2013, 25, 6874-6878.

8. $\quad$ Kozlovskaya, V.; Kharlampieva, E.; Mansfield, M. L.; Sukhishvili, S. A. Poly (methacrylic acid) Hydrogel Films and Capsules: Response to $\mathrm{pH}$ and Ionic Strength, and Encapsulation of Macromolecules. Chem.Mater. 2006, 18, 328-336.

9. $\quad$ Loretta, L.; Rivera-Gil, P.; Abbasi, A. Z.; Ochs, M.; Ganas, C.; Zins, I.; Sönnichsen, C.; Parak, W. J. LbL Multilayer Capsules: Recent Progress and Future Outlook for Their Use in Life Sciences. Nanoscale 2010, 2, 458-467.

10. Richardson, J. J.; Ejima, H.; Lörcher, S. L.; Liang, K.; Senn, P.; Cui, J.; Caruso, F. Preparation of Nano-and Microcapsules by Electrophoretic Polymer Assembly. Angew. Chem. Int. Ed. 2013, 52, 6455-6458.

11. Tong, W.; Gao, C.; Möhwald, H. Single Polyelectrolyte Microcapsules Fabricated by Glutaraldehyde-Mediated Covalent Layer-by-Layer Assembly. Macromol. Rapid Commun. 2006, 27, 2078-2083.

12. Wang, Z.; Zhu, H.; Li, D.; Yang, X. Preparation and Application of Single Polyelectrolyte Microcapsules Possessing Tunable Autofluorescent Properties. Colloids Surf., A 2008, 329, 58-66.

13. Vidyasagar, A.; Sung, C.; Gamble, R.; Lutkenhaus, J. L. Thermal Transitions in Dry and Hydrated Layer-by-Layer Assemblies Exhibiting Linear and Exponential Growth. ACS Nano 2012, 6, 6174-6184.

14. Mueller, R.; Köhler, K.; Weinkamer, R.; Sukhorukov, G.; Fery, A. Melting of PDADMAC/PSS Capsules Investigated with AFM Force Spectroscopy. Macromolecules 2005, 38, 9766-9771.

15. Jang, W.-S.; Jensen, A. T.; Lutkenhaus, J. L. Confinement Effects on Cross-Linking within Electrostatic Layer-by-Layer Assemblies Containing Poly(allylamine hydrochloride) and Poly(acrylic acid). Macromolecules 2010, 43, 9473-9479.

16. Richardson, J. J.; Björnmalm, M.; Gunawan, S. T.; Guo, J.; Liang, K.; Tardy, B.; Sekiguchi, S.; Noi, K. F.; Cui, J.; Ejima, H.; Caruso, F. Convective Polymer Assembly for the Deposition of Nanostructures and Polymer Thin Films on Immobilized Particles. Nanoscale 2014, 6, 13416-13420.

17. Ejima, H.; Yanai, N.; Best, J. P.; Sindoro, M.; Granick, S.; Caruso, F. NearIncompressible Faceted Polymer Microcapsules from Metal-Organic Framework Templates. Adv. Mater. 2013, 25, 5767-5771.

18. Moya, S.; Dähne, L.; Voigt, A.; Leporatti, S.; Donath, E.; Möhwald, H. Polyelectrolyte Multilayer Capsules Templated on Biological Cells: Core Oxidation Influences Layer Chemistry. Colloids Surf., A 2001, 183, 27-40.

19. Köhler, K.; Sukhorukov, G. B. Heat Treatment of Polyelectrolyte Multilayer Capsules: A Versatile Method for Encapsulation. Adv. Funct. Mater. 2007, 17, 2053-2061.

20. Déjugnat, C.; Köhler, K.; Dubois, M.; Sukhorukov, G. B.; Möhwald, H.; Zemb, T.; Guttmann, P. Membrane Densification of Heated Polyelectrolyte Multilayer Capsules Characterized by Soft X-ray Microscopy. Adv. Mater. 2007, 19, 1331-1336. 
21. Lvov, Y.; Decher, G.; Möhwald, H. Assembly, Structural Characterization, and Thermal Behavior of Layer-by-Layer Deposited Ultrathin Films of Poly(vinyl sulfate) and Poly(allylamine). Langmuir 1993, 9, 481-486.

22. Köhler, K.; Shchukin, D. G.; Möhwald, H.; Sukhorukov, G. B. Thermal Behavior of Polyelectrolyte Multilayer Microcapsules. 1. The Effect of Odd and Even Layer Number. $J$. Phys. Chem. B 2005, 109, 18250-18259.

23. Köhler, K.; Möhwald, H.; Sukhorukov, G. B. Thermal Behavior of Polyelectrolyte Multilayer Microcapsules: 2. Insight into Molecular Mechanisms for the PDADMAC/PSS System. J. Phys. Chem. B 2006, 110, 24002-24010.

24. $\quad$ Cui, J.; De Rose, R.; Alt, K.; Alcantara, S.; Paterson, B. M.; Liang, K.; Hu, M.;

Richardson, J. J.; Yan, Y.; Jeffery, C. M.; Price,R. I.; Peter, K.; Hagemeyer, C. E.; Donnelly, P. S.; Kent, S. J.; Caruso F. Engineering Poly(ethylene glycol) Particles for Improved Biodistribution. ACS Nano 2015, 9, 1571-1580.

25. Smith, R. N.; McCormick, M.; Barrett, C. J.; Reven, L.; Spiess, H. W. NMR Studies of PAH/PSS Polyelectrolyte Multilayers Adsorbed onto Silica. Macromolecules 2004, 37, 48304838.

26. Schwarz, B.; Schönhoff, M. A ${ }^{1}$ H NMR Relaxation Study of Hydration Water in Polyelectrolyte Mono and Multilayers Adsorbed to Colloidal Particles. Colloids Surf., A 2002, 198, 293-304.

27. Lo, M. Y.; Lay, C. L.; Lu, X.; Liu, Y. Finer Structures of Polyelectrolyte Multilayers Reflected by Solution 1H NMR. J. Phys. Chem. B 2008, 112, 13218-13224.

28. Richardson, J. J.; Maina, J. W.; Ejima, H.; Hu, M.; Guo, J.; Choy, M. Y.; Gunawan, S. T.; Lybaert, L.; Hagemeyer, C. E.; De Geest, B. G.; Caruso, F. Versatile Loading of Diverse Cargo into Functional Polymer Capsules. Adv. Sci. 2015, 2, 1400007.

29. Dong, W.-F.; Sukhorukov, G. B.; Möhwald, H. Enhanced Raman Imaging and Optical Spectra of Gold Nanoparticle Doped Microcapsules. Phys. Chem. Chem. Phys. 2003, 5, 30033012.

30. Dong, W.-F.; Ferri, J. K.; Adalsteinsson, T.; Schönhoff, M.; Sukhorukov, G. B.; Möhwald, H. Influence of Shell Structure on Stability, Integrity, and Mesh Size of Polyelectrolyte Capsules: Mechanism and Strategy for Improved Preparation. Chem. Mater. 2005, 17, 2603-2611.

31. Zucolotto, V.; Ferreira, M.; Cordeiro, M. R.; Constantino, C. J.; Balogh, D. T.; Zanatta, A. R.; Moreira, W. C.; Oliveira, O. N. Unusual Interactions Binding Iron Tetrasulfonated Phthalocyanine and Poly(allylamine hydrochloride) in Layer-by-Layer Films. J. Phys. Chem. B 2003, 107, 3733-3737.

32. Ge, L.; Pan, C.; Chen, H.; Wang, X.; Wang, C.; Gu, Z. The Fabrication of Hollow Multilayered Polyelectrolyte Fibrous Mats and Its Morphology Study. Colloids Surf., A 2007, 293, 272-277.

33. Zhao, H. C.; Wu, X. T.; Tian, W. W.; Ren, S. T. Synthesis and thermal property of poly (allylamine hydrochloride). Adv. Mater. Res. 2011, 150, 1480-1483.

34. Cornejo, J. J. M.; Matsuoka, E.; Daiguji, H. Size Control of Hollow Poly-Allylamine Hydrochloride/Poly-Sodium Styrene Sulfonate Microcapsules Using the Bubble Template Method. Soft Matter 2011, 7, 1897-1902.

35. Kolasińska, M.; Warszyński, P. The Effect of Support Material and Conditioning on Wettability of PAH/PSS Multilayer Films. Bioelectrochemistry 2005, 66, 65-70. 
36. Tenzer, S.; Docter, D.; Kuharev, J.; Musyanovych, A.; Fetz, V.; Hecht, R.; Schlenk, F.; Fischer, D.; Kiouptsi, K.; Reinhardt, C.; Landfester, K.; Schild, H.; Maskos, M.; Knauer, S. K.; Stauber, R. H. Rapid Formation of Plasma Protein Corona Critically Affects Nanoparticle Pathophysiology. Nat. Nanotechnol. 2013, 8, 772-781.

37. He, C.; Hu, Y.; Yin, L.; Tang, C.; Yin, C. Effects of Particle Size and Surface Charge on Cellular Uptake and Biodistribution of Polymeric Nanoparticles. Biomaterials 2010, 31, 36573666.

38. Hauck, T. S.; Ghazani, A. A.; Chan, W. C. Assessing the Effect of Surface Chemistry on Gold Nanorod Uptake, Toxicity, and Gene Expression in Mammalian Cells. Small 2008, 4, 153159.

39. Slowing, I.; Trewyn, B. G.; Lin, V. S.-Y. Effect of Surface Functionalization of MCM41-Type Mesoporous Silica Nanoparticles on the Endocytosis by Human Cancer Cells. J. Am. Chem. Soc. 2006, 128, 14792-14793.

40. Kai, M. P.; Keeler, A. W.; Perry, J. L.; Reuter, K. G.; Luft, J. C.; O'Neal, S. K.; Zamboni, W. C.; DeSimone, J. M. Evaluation of Drug Loading, Pharmacokinetic Behavior, and Toxicity of a Cisplatin-Containing Hydrogel Nanoparticle. J. Controlled Release 2015, 204, 70-77.

41. Poon, Z.; Lee, J. B.; Morton, S. W.; Hammond, P. T. Controlling In vivo Stability and Biodistribution in Electrostatically Assembled Nanoparticles for Systemic Delivery. Nano Lett. 2011, 11, 2096-2103.

42. Ladam, G.; Schaaf, P.; Cuisinier, F. J.; Decher, G.; Voegel, J.-C. Protein Adsorption onto Auto-Assembled Polyelectrolyte Films. Langmuir 2001, 17, 878-882. 
Table of Contents Graphic and Synopsis

Heat can be used as a driving force to reverse the charge of certain cationic polyelectrolyte films. This charge reversal allows for the subsequent addition of cationic polymers, and alternating polymer deposition and heat allows for layer-by-layer assembly. The heated films and core-shell particles also have lower biological interactions then unheated films.

J. J. Richardson, B. L. Tardy, H. Ejima, J. Guo, J. Cui, K. Liang, G. H. Choi, P. J. Yoo, B. G. De Geest, and F. Caruso*

Thermally Induced Charge Reversal of Layer-by-Layer Assembled Single-Component Polymer Films

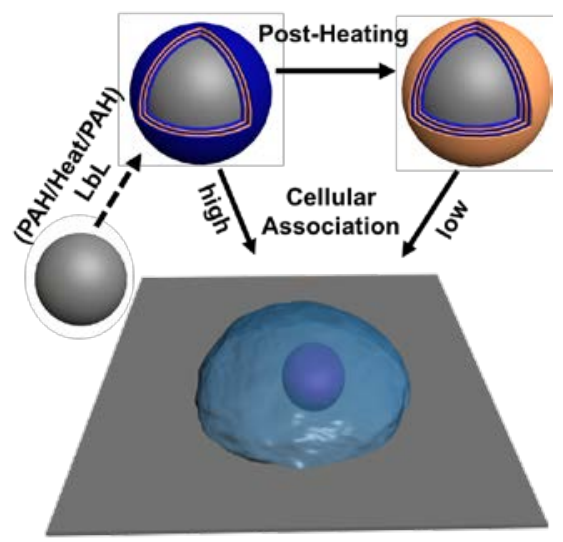




\section{University Library}

\section{- M M N E R VA A gateway to Melbourne's research publications}

Minerva Access is the Institutional Repository of The University of Melbourne

Author/s:

Richardson, JJ;Tardy, BL;Ejima, H;Guo, J;Cui, J;Liang, K;Choi, GH;Yoo, PJ;De Geest, BG;Caruso, F

Title:

Thermally Induced Charge Reversal of Layer-by-Layer Assembled Single-Component Polymer Films

Date:

2016-03-23

Citation:

Richardson, J. J., Tardy, B. L., Ejima, H., Guo, J., Cui, J., Liang, K., Choi, G. H., Yoo, P. J., De Geest, B. G. \& Caruso, F. (2016). Thermally Induced Charge Reversal of Layer-by-Layer Assembled Single-Component Polymer Films. ACS APPLIED MATERIALS \& INTERFACES, 8 (11), pp.7449-7455. https://doi.org/10.1021/acsami.6b00472.

Persistent Link:

http://hdl.handle.net/11343/111984 